\title{
„Unterstützte Erinnerung“ als Form der Wissensorganisation
}

\section{Zur Rolle der Fürsorgeakte in der biografischen Erinnerungsarbeit ehemaliger Heimkinder}

\author{
Flavia Guerrini · Ulrich Leitner • Michaela Ralser
}

Online publiziert: 3. Januar 2020

(C) Der/die Autor(en) 2019

Zusammenfassung Mündliche und schriftliche Quellen validieren sich nicht gegenseitig. Vielmehr - so argumentieren die AutorInnen - stellen sie Erkenntnismittel im je eigenen Recht dar. Was sich ereignet, wenn - wie in der Heimgeschichteforschung nicht unüblich - die schriftliche Quelle der Jugendfürsorgeakte von den Interviewten, den ehemaligen Heimkindern, selbst als Erkenntnismittel und Erinnerungsstütze genutzt wird, davon handelt der vorliegende Beitrag. Er zeigt die Bedeutung der Fürsorgeakte im Rahmen biografischer Erinnerungsarbeit - gerade für Personen, die aufgrund ihrer spezifischen Aufwachsensbedingungen in Fremdunterbringungsinstitutionen vom üblicherweise zur Verfügung stehenden generationalen Gedächtnis ausgeschlossen sind. Der Beitrag dokumentiert und diskutiert den Gebrauch dieser spezifischen schriftlichen Quelle als Organisatorin und Ordnerin biografischer Wissensbestände, als Mobilisatorin und Mediatorin erinnerter Erfahrung, aber eben auch als aufklärungsbedürftige Falschmeldung, Fehldeutung und Leerstelle. Er zeigt beispielhaft den herausfordernden, aber letztlich gelingenden Umgang einer Interviewpartnerin mit der über sie angelegten Fürsorgeakte und gibt darüber hinaus Auskunft über die darin aufgeworfenen Fragen nach Gedächtnis, Erinnern und Repräsentation.

F. Guerrini $(\bowtie) \cdot$ U. Leitner $\cdot$ M. Ralser

Institut für Erziehungswissenschaft, Universität Innsbruck, Liebeneggstr. 8, 6020 Innsbruck,

Österreich

E-Mail: flavia.guerrini@uibk.ac.at

U. Leitner

E-Mail: ulrich.leitner@uibk.ac.at

M. Ralser

E-Mail: michaela.ralser@uibk.ac.at 


\title{
“Supported Memory" as a Form of Knowledge Organization
}

On the Role of Child Welfare Case Files in the Biographical Memory Work of Care Leavers

\begin{abstract}
Oral and written sources do not validate each other. Rather, as the authors will argue, they constitute means of knowledge in their own right. This paper will deal with a specific albeit not uncommon constellation in the research on history of residential child care and pose the question what happens when care leavers use their case files as a means of insight and a memory aid. The authors aim to highlight the meaning of child care case files in the context of biographical memory work. This is especially the case for care leavers who don't have access to the usually available inter-generational memory due to growing up in residential care institutions. The paper documents and discusses the use of this specific written source in order to organize and arrange biographical knowledge and to mobilize and mediate remembered experience. Misrepresentations, misinterpretations and omissions might simultaneously occur and become apparent. By way of example, this paper shows the challenging but ultimately successful way one interviewee used to deal with her own case file, and also provides information on the questions of memory, remembrance and representation.
\end{abstract}

\section{Einleitung}

Das Jahr 2010 markiert das Anheben einer neuen Aufmerksamkeit für die unterschiedlichen Formen körperlicher, psychischer, sexualisierter und struktureller Gewalt, denen Kinder und Jugendliche in Kinder- und Erziehungsheimen bis in die jüngere Vergangenheit ausgesetzt waren. Seither wurden in Deutschland, Österreich und der Schweiz Opferschutzstellen, Runde Tische und ExpertInnenkommissionen eingerichtet, PolitikerInnen und VertreterInnen von privaten Heimträgern sprachen öffentliche Entschuldigungen aus, in einigen Ländern wurden Entschädigungsleistungen für ehemalige Heimkinder und andere Opfer fürsorgerischer Zwangsmaßnahmen bereitgestellt. Parallel dazu entstand eine Vielzahl an teils von öffentlicher Hand in Auftrag gegebenen, teils über die Einwerbung von Drittmitteln finanzierten, oder auch im Rahmen von Qualifikationsarbeiten durchgeführten wissenschaftlichen Untersuchungen, mit dem Ziel, die bislang wenig beachtete Geschichte der Heimerziehung und Fremdunterbringung zu rekonstruieren und die Erfahrungen der von dieser jüngeren Geschichte Betroffenen in das öffentliche Gedächtnis einzuschreiben. Aufgrund der spezifischen Beschaffenheit des überlieferten Schriftguts zur Heimgeschichte, das zum größeren Teil als obrigkeitliche Quelle zu charakterisieren ist (Ralser 2010, S. 178), aber auch wegen oft erheblicher Lücken in der Überlieferung der zeitgenössisch angefertigten Aufzeichnungen und Akten kommt der Erinnerung von ZeitzeugInnen besondere Bedeutung zu.

Die forschungsseitigen Bemühungen der Rekonstruktion und Einordnung der Geschichte der Heimerziehung finden ihre Entsprechung in den Bemühungen der ZeitzeugInnen, sich ihre eigene Vergangenheit zu erschließen und ihr Sinn zu verleihen. Das etwa wird im Fall der Zeitzeugin Jutta Vierthaler (Pseudonym) deutlich, die 
wir als Interviewpartnerin für ein Forschungsprojekt zur Geschichte der westösterreichischen Jugendfürsorge mit dem Titel „Regime der Fürsorge. Geschichte der Heimerziehung in Tirol und Vorarlberg (1945-1990)“ gewinnen konnten (Ralser et al. 2017 und Universität Innsbruck 2019). Jutta Vierthaler war Anfang der 1950er Jahre von ihrem vierten bis zu ihrem siebten Lebensjahr in einem städtischen Kinderheim in Innsbruck untergebracht. Nachdem seit 2010 auch in Westösterreich die Archive geöffnet wurden, forderte sie als eine der ersten der ehemaligen Heimkinder aus Tirol Einsicht in ihre Fürsorgeakte. Im August 2013 meldete sich Vierthaler auf den Aufruf des Forschungsprojektes „Regime der Fürsorge“, um als Zeitzeugin daran mitzuwirken. Aus den frühen Jahren ihrer Kindheit ist ein Foto von ihr und ihrem Bruder erhalten, das Jutta Vierthaler zum Interviewtermin mitbrachte: Es zeigt sie selbst und den Buben Hand in Hand und herausgeputzt, bei einem angekündigten Besuch der „Obrigkeit“, wie sie sagte. Nie seien sie sonst so warm angezogen und nie so glücklich gewesen, wie es auf dem Bild den Anschein macht. Neben diesem Bild ist die Akte, die im Stadtjugendamt Innsbruck über sie angelegt wurde, das einzige Erinnerungsstück, das ihr von der Kinderzeit geblieben ist. Als sie die Akte in den Räumen der Opferschutzstelle Tirol zum ersten Mal zu lesen begonnen hatte, ging es ihr sehr schlecht, erzählte sie im Interview. Man habe ihr psychologische Betreuung angeboten, aber sie wollte es zuerst einmal allein versuchen. Sie merkte schnell, so sagte sie, dass sie die Akte nicht in einem Stück würde lesen können und blätterte sie erst einmal mehrmals durch, um zu sehen, was sie überhaupt enthielt. Den Moment des ersten Lesens und den weiteren Umgang mit der Akte schildert Jutta Vierthaler folgendermaßen:

[Ich] bin dann ziemlich schnell draufgekommen, dass da sehr wenig von dem drin ist, was ich eigentlich gewünscht hätte. Andererseits kann man natürlich nicht erwarten, dass sie die ganzen Prügelorgien, die sie da aufgeführt haben, da festhalten drin, ist auch klar. Aber es hat mir ein Stück Vergangenheit zurückgebracht [...] Ich habe dann ja immer wieder einmal so [an] einem Abend, wo es mir am Tag gut gegangen ist, [...] wieder hineingeschaut und teilweise habe ich eigentlich das eher emotionslos lesen können und teilweise ist es dann wirklich schlimm geworden. Es haben sich dann auch natürlich meine Albträume wieder eingestellt. Und was mich selbst überrascht hat: Dass erst mit der Zeit dann so viele Details wieder daher gekommen sind für mich. Eigentlich Details, wo ich bei den Therapien mir gedacht habe, wenn, dann kommt es spätestens dort, [aber] das war gar nicht so. [...] Sondern: Zuerst waren so Bruchstücke da [...] zuerst gehen die Bilder ganz schnell und dann bleiben sie plötzlich teilweise stehen und das ist seit ich den Akt jetzt daheim habe, passiert mir das. Ich habe es davor erfolgreich [...] verdrängt: Ich bin eigentlich dem Ganzen davongerannt, bin ich später draufgekommen. (Vierthaler 2013b, 01:32-01:34)

In dieser Interviewsequenz spricht die Zeitzeugin einige Aspekte des komplexen Verhältnisses von biographischer Erinnerung und schriftlicher Überlieferung an, die hier in der sehr spezifischen Form der Jugendfürsorgeakte vorliegt: Zunächst enttäuscht die Akte die Erwartungen der Zeitzeugin, nämlich eine Bestätigung der widerfahrenen Gewalt zu erhalten. Durch das Wachrufen von Erinnerungen ermög- 
licht sie andererseits eine Annäherung an ihre Kindheit, die ihr zuvor nicht möglich war. Nicht einmal in der Psychotherapie seien - so meint sie - diese Erinnerungen zum Vorschein gekommen. Die Lektüre der eigenen Akte verweist somit auf die Lückenhaftigkeit sowohl der Erinnerung als auch der schriftlichen Aufzeichnungen. Angesprochen werden aber auch die emotionalen Reaktionen und die Eigendynamik der aufsteigenden Erinnerungen, die die Auseinandersetzung der Zeitzeugin mit der über sie angelegten Akte in Gang gesetzt hat. Wie Jutta Vierthaler haben auch andere ZeitzeugInnen ihre eigenen Akten - das heißt, ihre Zöglingsakte aus einem Erziehungsheim oder die Fallakte des zuständigen Jugendamtes - ausgehoben und sich intensiv mit ihnen befasst. Sie zogen die von den Protagonisten und Protagonistinnen der Jugendfürsorgebehörden und -institutionen verfassten Akten zur Aufklärung der eigenen Vergangenheit heran. Der Blick auf die vermeintlich ,verwahrlosten“ Kinder (vgl. etwa Ramsauer 2000), der in diesen Schriftstücken dokumentiert ist, steht allerdings, so vermittelten es die Aussagen der InterviewpartnerInnen, häufig in Kontrast zu deren Erfahrungen und Deutungen.

Der vorliegende Beitrag versucht nun Einblicke in die komplexe, in der Interviewsituation mit ehemaligen Heimkindern auftretende, Gemengelage zwischen biographischer Erinnerung und schriftlicher Überlieferung zu geben. In dieser spezifischen Konstellation tritt die interviewte Person nicht nur mit der Interviewerin bzw. dem Interviewer in Interaktion, sondern gleichsam auch mit ihrer Fürsorgeakte. Der Beitrag will damit auf einen Aspekt der Beziehung von Menschen zu Verwaltungsakten aufmerksam machen, der in narrativen Interviews mit Personen, die über Kenntnis der über sie angefertigten Akten verfügen, zu Tage tritt. Er will das Verhältnis der Konstruktion der adressierten Individuen im Zuge bürokratischen Schrifthandelns (Foucault 1976, S. 246) und der biographischen Selbstkonstruktion der betroffenen Menschen an einem Beispielfall beschreiben und diskutieren. Dazu soll im Folgenden zunächst der Forschungshintergrund vorgestellt werden, in den die im Beitrag verwendeten Hauptquellen, das narrativ-biographische Interview mit Jutta Vierthaler und ihre Fürsorgeakte, eingebettet sind. Sodann wird die individuelle und kollektive Erinnerungsarbeit im spezifischen Kontext der Heimgeschichte und ihrer neuen ZeitzeugInnenschaften und Aufmerksamkeitsökonomien dargestellt, um im Anschluss am Beispiel der Zeitzeugin Jutta Vierthaler die Fürsorgeakte als spezifische Erinnerungsstütze im narrativen Interviewsetting vorzustellen. Dabei werden drei Formen der Bezugnahme der Zeitzeugin zur Akte herausgearbeitet. Im abschlieBenden Fazit wird der Umgang mit Fürsorgeakten im Kontext von Erinnerungs- und Verarbeitungsprozessen der Heimgeschichte zusammenfassend eingeholt und zu allgemeineren gedächtnistheoretischen Fragestellungen hin geöffnet.

\section{Das Forschungsprojekt: Regime der Fürsorge}

Das Forschungsprojekt, in dessen Rahmen das biographische Interview mit der Zeitzeugin Jutta Vierthaler geführt wurde, gehört zu jenen, die in den 2010er Jahren von öffentlichen Trägern der ehemaligen Fürsorgeerziehungsheime - hier den österreichischen Bundesländern Tirol und Vorarlberg - in Auftrag gegeben wurden. Ziel war es, die Geschichte des Fürsorgeerziehungssystems in Westösterreich sowie seiner 
vier öffentlichen Landeserziehungsheime zu erarbeiten, daraus Schlüsse hinsichtlich der wesentlichen Akteure, Kräfte und Verantwortlichkeiten zu ziehen und eine vertiefte Kenntnis über die Zusammenhänge, welche die historische Kinder- und Jugendhilfe der Region kennzeichneten, zu gewinnen, zu vermitteln und vor dem Hintergrund der jeweiligen Zeit sozial-, diskurs- und geschlechtertheoretisch zu interpretieren. Dabei sollten das Fürsorgeerziehungssystem, die Fürsorgeerziehungseinrichtungen und die Wirkungen, welche sie in den Kindern und Jugendlichen hinterlassen haben, so präzise wie möglich beschrieben und eingeordnet werden. Dazu wurde ein multiperspektivischer Zugang zur Geschichte über zumindest drei Informationsquellen gewählt (vgl. Ralser et al. 2017, S. 33 ff.):

1. Die heterogenste Quellensorte stellte das allgemeine überlieferte Schriftgut dar: Texturen aller Art, Dokumente und Bilder, Chroniken, Landtagsprotokolle und Kontrollberichte, Zeitungsbeiträge, welche Auskunft über die zeitgenössische Öffentlichkeit der Heimerziehung und damit Einblick in den je historischen Wissensund Debattenstand ermöglichen sowie die historisch spezifische Mitwisserschaft politisch-administrativer, aber auch zivilgesellschaftlicher AkteurInnen kenntlich machen.

2. Die zweite Quellenart ist das überlieferte Aktenschriftgut des Fürsorgeerziehungssystems, seiner Apparate, Agenturen und Einrichtungen selbst. Von den unterschiedlichen sach- und personenbezogenen Akten erwiesen sich die sogenannten Mündel- und Zöglingsakten als besonders interessante Quellen (für einen Überblick zu den personenbezogenen Akten vgl. Ralser et al. 2014, S. 56-99). Als wesentliche Medien der Kommunikation, als „Gedächtnis der Verwaltung [zur] Sicherung kontinuierlicher Handlungsmuster" (Müller und Müller 1984, S. 23) in arbeitsteiligen Verwaltungsabläufen, hier der Verwaltung der unter Fürsorgeerziehung stehenden Kinder und Jugendlichen, geben sie über die in den jeweiligen Institutionen etablierten Deutungsmuster und -routinen Auskunft. Sie zeigen den sogenannten Aktenzögling (Zaft 2011) und das obrigkeitliche Wissen, das ihn hervorbringt, geben zeittypische Normalitätsvorstellungen preis, lassen Einweisungs-, Versetzungs- und Entlassungsprozeduren rekonstruieren und machen nachvollziehbar, dass diese spezifische Akte in ihrer Wirkung auf die Lebensverläufe und Lebenschancen der befürsorgten Kinder über die Gedächtnisfunktion in administrativen Zusammenhängen deutlich hinausgeht (vgl. Guerrini 2018).

3. Die dritte Quellenart ist das biografisch-narrative Interview (Schütze 1983) in der Forschungstradition und -perspektive der Oral History (Niethammer 1980; Wierling 2003). Aus erkenntnistheoretischer Sicht lässt es ,einen umfassenderen und in sich strukturierteren Zugang zur Erfahrungswelt“ (Flick 2009, S. 227) der befragten Personen sowie mehr Offenheit für nicht bereits vorab Erwartetes und Vermutetes zu und macht damit auch nicht-explizierbares, latentes Wissen der Rekonstruktion zugänglich (vgl. Rosenthal 2008, Lucius-Hoene und Deppermann 2004). Durch eine erzählgenerierende Eröffnungsfrage soll die Auskunft gebende Person zu einer spontanen, freien, autobiografischen Erzählung angeregt werden, die durch die Wirksamkeit der sogenannten Erzählzwänge (Gestaltschließungs-, Kondensierungs- und Detaillierungszwang) eine besondere Ausgewogenheit an 
Detailliertheit, tiefgründigen Einblicken und forschungspragmatischer Kompaktheit erhält (Rosenthal 2008, S. 141). Mit 92 ZeitzeugInnen waren wir in Kontakt, mit 48 konnten wir ein Interview führen: der allergrößte Teil mit ehemals in Heimen untergebrachten Menschen, die zum Zeitpunkt der Interviews zwischen Mitte 40 und nahezu 90 Jahre alt waren, einige mit früherem pädagogischen Personal sowie weiteren relevanten Auskunftspersonen. Der Großteil der Befragten war in den 1950er bis 1970er Jahren in den Heimen der Region untergebracht worden, die Zeit, in der auch die größte Dichte der Heimeinweisungen zu verzeichnen ist (Ralser et al. 2017, S. 62-74).

\section{Erinnerung und ZeugInnenschaft im Kontext der Heimgeschichte}

Unterschiedliche Daten, hier die aus den ZeitzeugInneninterviews gewonnenen auf der einen und die aus der historische Aktenanalyse rekonstruierten auf der anderen Seite, validieren sich nicht umstandslos wechselseitig (vgl. zu letzterem: Bereswill und Rieker 2017). Dennoch aber stellen beide Quellen wichtige Erkenntnismittel dar und erzeugen letztlich Erkenntnisse im je eigenen Recht, sodass von einem doppelten Vetorecht dieser beiden Quellensorten gesprochen werden kann: Dem Vetorecht der mündlichen Quelle und dem Vetorecht der Akte gegenüber dem kollektiven Gedächtnis einerseits und von doppelter Geschichts- und Wahrheitsproduktion andererseits (Ralser et al. 2017, S. 63). Akten und Interviews erzählen unterschiedliche Geschichten. Der Quellenkritik bedürfen beide. Aus historischen Fallakten der Fürsorgeerziehung kann der herrschaftskonstituierende Blick auf vermeintlich verwahrloste Kindheiten rekonstruiert werden, aus den Interviews das subjektive Erleben der Heimerziehung vor dem biografischen Hintergrund der ZeitzeugInnen und in Relation zu ihrer individuellen wie kollektiven Gegenwart. Das Wissen um die jeweils andere Erzählung orientiert und bedingt die spezifische Lesbarkeit der Quellen durch die Wissenschaft, aber auch durch die Betroffenen selbst, wie am Beispiel Jutta Vierthaler im Folgenden noch ausführlich gezeigt werden wird.

Wie schon das Erinnern an die jeweilige Heimgeschichte und das Abfragen dieser Erinnerung ist auch die ZeitzeugInnenschaft kontextrelational. Um die Heimkindheit der InterviewpartnerInnen nicht überzudeterminieren, so als hätte es kein erzählenswertes Leben davor oder danach gegeben, haben wir sie in den Zeitungsaufrufen, den schriftlichen Anfragen und den Bereitschaftserklärungen nicht als ehemalige Heimkinder adressiert, wie es manche von ihnen selbst taten, sondern als ZeugInnen ihrer Zeit angesprochen. Dass wir mit der ZeitzeugInnenschaft selbst ein spezifisches Konzept aufgerufen hatten (vgl. Sabrow 2012), war uns vorerst nicht bewusst. Diese Benennung markiert eine zeitspezifische Transformation, die sowohl die Forschung, wie die Erinnerung und die SprecherInnenpositionen veränderte. Nicht nur war die Forschung zur regionalen Heimgeschichte als Beitrag zur Aufklärung über die Verhältnisse der öffentlichen Ersatzerziehung nach 1945 positioniert, sie wurde auch von der historischen Zeit der 2010er Folgejahre so eingeordnet. Die Forschungen fanden in einer Zeit erhöhter politischer und medialer Aufmerksamkeit für das Thema statt: flankiert von Weltnachrichten über Missbrauch in pädagogischen Institutionen, den Ausläufern der Debatten um den Runden Tisch Heimerziehung in 
Deutschland sowie nationaler und vor allem lokaler medialer Berichterstattung über die Gewalt in den Erziehungsheimen der Region, mit oft markigem Vokabular, drastischer Klage und im Gestus heroischer Geschichtsaufklärung (u. a. Schreiber 2010; Hönigsberger und Karlsson 2013).

In den Ländern und Diözesen wurden Opferschutzkommissionen eingerichtet, die die den Heimkindern widerfahrene Gewalt als illegitim einzuschätzen begannen, mit diesen ihre Akten aushoben und Entschädigungsverfahren einzuleiten bereit waren. Bislang ungehörte Geschichten erlangten den Status repräsentativer ZeugInnenschaft. Die lange ungehörte Klage der Betroffenen hatte sich vor diesem Hintergrund zu bewähren und scheiterte oftmals im öffentlichen Auftritt. Wie überhaupt die öffentlichen Auftritte mehrfach prekär wurden, weil sie nahezu unentrinnbare Rollengefäße bereithielten: Diese riefen die sich Erinnernden als ZeugInnen einer Vergangenheit auf, setzten sie als GeschichtsvermittlerInnen ein, begünstigten heroische Figuren und bestimmten bald, was von wem zu erinnern war und warum. Darin erlangten auch die ForscherInnen eine neue, nicht immer freiwillige Rolle: als Stimme, als Sprachrohr, als StreiterInnen für das „Gerechte“ und die „Geschädigten“. Der Anspruch ,,jetzt reden wir“ (Heimkinder reden 2015) wurde so auch zunehmend ambivalent: Er veränderte zwar die SprecherInnenpositionen grundlegend, erzeugte gleichzeitig aber neue, autorisierte und weniger autorisierte ZeugInnen des Vergangenen. Dies alles beeinflusste als soziale Gegenwartsbedingung auch die biografischnarrativen Interviews. Diese aber bergen gegenüber der medial-öffentlichen ZeitzeugInnenschaft, die als ,kulturelle Pathosformel“ (Aby Moritz Warburg) auch die Aufmerksamkeitsökonomie der Heimgeschichte der Region zu bestimmen begann, den unhintergehbaren Vorteil, dass sie eben genau dieses Ringen mit der sozialen Rahmung ihres Erinnerns preisgeben und für die sich Erinnernden ebenso wie für die ihnen Zuhörenden einseh- und verhandelbar machen (vgl. Sieder und Smioski 2012). Dafür, wie deutlich die Gegenwartsbedingungen die SprecherInnenpositionen der InterviewpartnerInnen bestimmten und ihre ZeugenInnenschaft einordneten, sollen hier nur einige wenige Beispiele aus den Beginnsequenzen von Kontaktaufnahmen und Interviews aus dem Forschungsprojekt genannt werden: „Ich rede hier bei Ihnen auch für die vielen, die das nicht mehr können“ und ,gekommen bin ich, weil, weil - damit das nie wieder passiert“ oder „Ich hab's im Radio gehört - den Aufruf, da habe ich mir gedacht, ich muss mich gleich bei Ihnen melden und da bin ich“; oder auch „Ich war zwar im Heim aber bei mir war's nicht so schlimm“ und „,Der P., der hat mich nicht genommen [für sein Forschungsprojekt, Anm.d.V.], wahrscheinlich hat ihm meine Geschichte nicht gepasst."

Erinnern in all seinen Formen - darüber herrscht inzwischen Einigkeit - dient dazu, die vergangenen Erfahrungen in der aktuellen Situation und auch im Hinblick auf die Zukunft einzuordnen und $\mathrm{zu}$ interpretieren. In einem umfassenderen Sinn verhilft das Erinnern dem Menschen zur Biografie. Der als Zeitzeuge Adressierte übernimmt mehr noch als der in Alltagssituationen sich Erinnernde von der Vergangenheit die Erinnerung, von der Gegenwart aber die Wertmaßstäbe, das kulturelle Rahmenformat, in dem er das Vergangene memoriert und zugleich aktualisiert (vgl. Sabrow et al. 2007). Das heißt auch, dass die Aussagen der ZeitzeugInnen der Heimgeschichte institutionell eingebettete dialogische Sprechakte darstellen, die erst im Rahmen eines komplexen sozialen Netzes, kodifizierter Rituale und Konventionen zu 
Zeugenaussagen werden und als solche Gehör finden. Die ehemaligen Heimkinder sind - mit Ausnahme einer sehr kurzen Phase in den 1970ern - erst vergleichsweise spät als ZeugInnen ihrer Geschichte anerkannt worden. Sie haben beispielsweise in Österreich erst seit den 2010er Jahren eine legitime SprecherInnenposition erlangt. Entscheidend dafür waren eine aufmerksame Öffentlichkeit, eine Aufnahme des Themas in die politisch-parlamentarische Debatte, publikumswirksame journalistische Publikationen einschließlich der Selbstbeschreibungen ehemaliger Heimkinder und wissenschaftliche Arbeiten, die ZeitzeugInnenberichte zentral einbezogen und ihnen darüber soziale Geltung verschafften. Spätestens seit der breiteren Rezeption der Überlegungen zum kollektiven und kulturellen Gedächtnis (vgl. Halbwachs 1985 [1925]; Assmann 1996; Assmann 2007) wissen wir um die soziale Rahmung auch der individuellen Erinnerung, um die Verwobenheit der eigenen Erinnerung mit der anderer, um ihre Strukturierung durch Erzählung und Wiedererzählung, schließlich um ihren kommunikativen Herstellungsmodus im Rahmen geteilter Erfahrung. Selbstredend stehen die individuellen und Gruppenerinnerungen in den ZeitzeugInneninterviews auch in einer spezifischen Korrespondenz mit dem sogenannten kollektiven Gedächtnis, das sich in seiner allgemeinsten Form als an feste Objektivationen gebundene, hochgradig gestiftete und ritualisierte vergegenwärtigte Erinnerung beschreiben ließe und auch die offizielle Geschichtsschreibung miteinschließt (Assmann 2007). Gerade jene Menschen, die einen größeren Teil ihrer Kindheit und Jugend in staatlichen Institutionen verbracht haben, sind in ihrer Erinnerungsfähigkeit auf besondere Weise davon abhängig, sich als Erwachsene ein kohärentes Bild über sich als Kinder und Jugendliche zu machen, zumal ihre spezifische biografische Konstellation, das Aufwachsen außerhalb elterlicher oder familialer Strukturen, sie vom generationalen Gedächtnis ebenso abschließt, wie von den vielfältigen Erinnerungsstützen, die Eltern ihren Kindern durch Erzählung beständig zur Verfügung stellen.

Hinzu kommt noch ein weiteres Spezifikum, zumindest für einige der ZeitzeugInnen der Heimgeschichte, nämlich dass ihre Sprechakte von der in die Gegenwart fortdauernden, schmerzlichen Präsenz der Vergangenheit unterbrochen und aus dem Takt gebracht werden (vgl. Brunner 2012), dass das Fragmentarische, Episodische und Unzuverlässige, das jedes Erinnern kennzeichnet, noch deutlicher hervortritt und eine kohärente Erzählung erschwert. Dass gerade hier, aber längst nicht nur hier, aus den kollektiven Erzählungen entnommene Ersatzbilder und vorgefertigten Erzählgefäße zum Einsatz kommen, aber auch Erinnerungsstützen aller Art, verwundert nicht: vom Rekurs auf einen berufeneren Erzähler, der alles schon gesagt hätte, der wörtlichen Wiederholung einmal gefundener und beibehaltener Narrationssequenzen, von der mitgebrachten Gedächtnisnotiz und anderen autobiografischen Texturen bis hin zu fremdgefertigten Dokumenten über die Erzählenden, etwa die ausgehobene, eigene Jugendwohlfahrtsakte. Es ist ein Vorteil gerade des biografisch-narrativen Interviews und seiner Anwendung im Kontext von Forschungen zur Sozialisation in Zwangsinstitutionen, dass diese Prozesse nicht entgehen, sondern sichtbar bleiben und interpretierbar werden. 


\section{Die Akte als Erinnerungsstütze in der biographischen Selbstkonstruktion}

Jutta Vierthalers Erinnerungsarbeit verdeutlicht, dass biographisches Erzählen für den Aufbau der jeweils eigenen Lebensgeschichte und insbesondere für Sinngebungsprozesse hinsichtlich der gemachten Erfahrungen von Bedeutung ist. Stellvertretend für viele andere ehemalige Heimkinder führt das Interview mit ihr vor Augen, unter welch erschwerten Bedingungen die InterviewpartnerInnen diese Biographiearbeit zu leisten haben. Kinder erlernen biographisches Erinnern und Erzählen meist durch gemeinsames Erinnern und Erzählen mit ihren elterlichen Bezugspersonen und bauen im Zuge dessen ab der frühen Kindheit ein autobiographisches Gedächtnis auf (Fivush und Hessel 2010. S. $47 \mathrm{ff}$.). Vor allem bei früh beginnenden und lange andauernden Heimaufenthalten, aber auch in unsteten, durch Beziehungsabbrüche oder unterschiedliche Formen von Gewalt gekennzeichneten Familienkonstellationen fehlt diese Praxis des gemeinsamen Erinnerns und Erzählens. Das trifft auf Jutta Vierthaler zu: Sie und ihr älterer - mittlerweile verstorbener - Bruder Eberhard wurden in schwierige familiäre Verhältnisse hineingeboren. Die noch sehr junge Mutter bekam die beiden Kinder im Abstand von nur 10 Monaten. Alle drei waren der Gewalt des alkoholkranken Vaters und der Kontrolle seiner als autoritär beschriebenen Mutter ausgesetzt. Die beiden Kinder kamen im Alter von etwa 31/2 und 41/2 Jahren aufgrund der Delogierung der Eltern ,,vorübergehend“ - wie es laut Akte geplant war - in ein städtisches Kinderheim in Innsbruck, wo sie laut den Aussagen der Zeitzeugin andauernder körperlicher wie psychischer Gewaltanwendung sowohl durch das Personal als auch durch ältere Heimkinder ausgesetzt waren. Aus der als Übergangslösung gedachten Heimunterbringung wurde schließlich ein über drei Jahre dauernder Aufenthalt.

Die frühe Heimeinweisung, die lange Dauer der anstaltsförmigen Unterbringung und der weitgehend monotone Alltag können dazu beigetragen haben, dass viele Befragte über vergleichsweise wenige Erinnerungen an ihre Kindheit in Heimen verfügen (Kuhlmann 2008, S. 124). Ein gemeinsames Erinnern an die Kindheit und Jugend kann auch häufig zu einem späteren Zeitpunkt im Leben nicht ausreichend nachgeholt werden, da bei vielen Betroffenen Auskunftspersonen sowie Medien der Erinnerung wie Fotos, Briefe oder Gegenstände aus der Kindheit fehlen oder nur eingeschränkt zur Verfügung stehen. Schließlich ist das Leben von nicht wenigen ehemals in Heimen untergebrachten Menschen aus verschiedenen Gründen durch lange Phasen des Nicht-Erinnerns, Verdrängens, Schweigens gekennzeichnet - sei es aus Scham, aufgrund erlittener Traumatisierungen oder schlichtweg, weil keine ZuhörerInnen zur Verfügung stehen. Jutta Vierthaler griff in dieser Situation auf ihre Fürsorgeakte zur Gestaltung ihres Erinnerungsprozesses zurück. Im Folgenden sollen drei Aspekte der Bezugnahme auf die Akte im Erinnerungsprozess Jutta Vierthalers vorgestellt werden. 


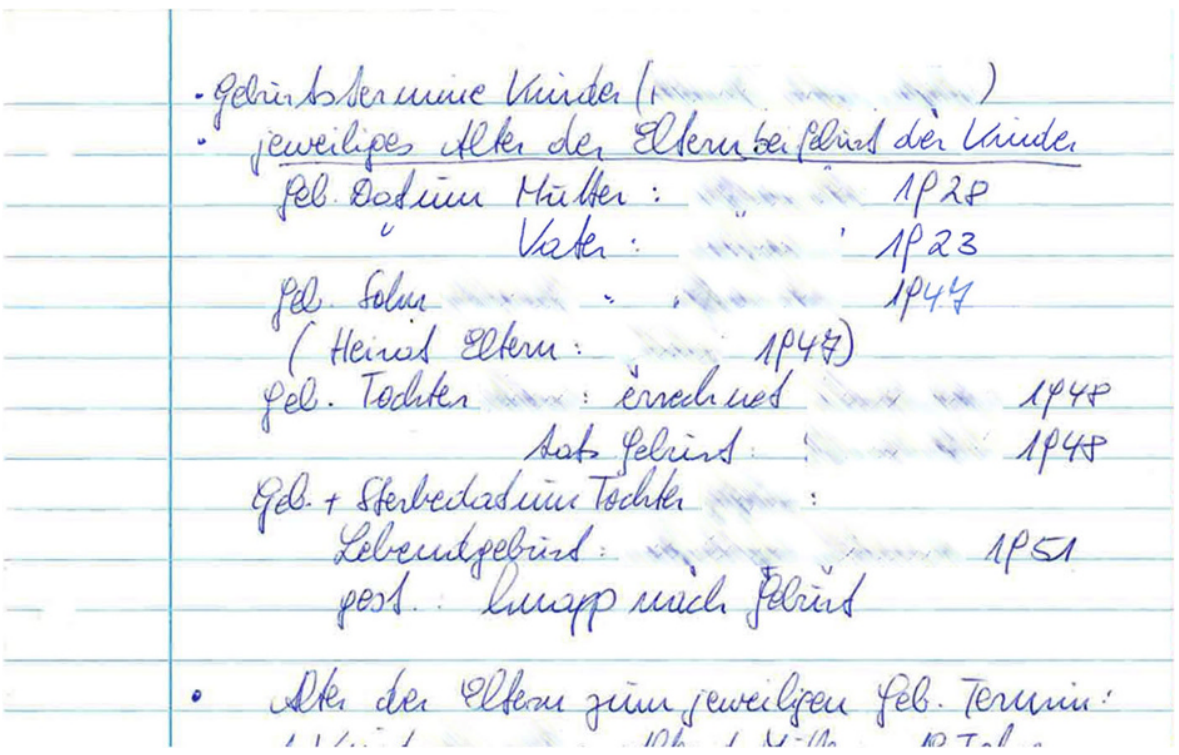

Abb. 1 Ausschnitt aus Jutta Vierthalers „Daten/Zusammenfassung“ (Quelle archiviert am Institut für Erziehungswissenschaft der Universität Innsbruck): Die Akte wird zur Rekonstruktion und Verarbeitung wichtiger Lebensdaten herangezogen

\subsection{Anreicherung der biographischen Narration durch Informationen aus der Akte}

Eine erste Verwendung findet die eigene Akte zur Rekonstruktion fehlender Informationen hinsichtlich des eigenen Lebens. Jutta Vierthalers Bedürfnis, ihrer Vergangenheit eine Ordnung zu geben, ist u. a. in einem selbst angefertigten, teils handschriftlich und teils am Computer verfassten vierseitigen Schriftstück zu erkennen. Es trägt den Titel „Daten/Zusammenfassung zu den diversen Schriftstücken“ (Vierthaler „Daten/Zusammenfassung“, siehe Abb. 1) und wurde in der dem Projekt überlassenen Mappe den Aktenkopien gleichsam vorangestellt. Es gibt Auskunft über die von der Zeitzeugin als relevant erachteten Ereignisse in der Kindheit und Jugend: Geburtsdaten von Familienmitgliedern, Eheschließungen, Scheidungen, Wiederverheiratung, Daten zu den Wohnungen der Familie, zur Lehre etc., enthält aber auch Beschreibungen und erinnerte Episoden zu den benannten Abschnitten ihrer Kindheit und Jugend.

Die Bedeutung, die der Akte zum Erlangen von Informationen über die eigene Kindheit zukommt, zeigt sich auch vor dem Hintergrund, dass Jutta Vierthaler ganz wesentliche Informationen zu ihrer Familie fehlten. Im Interview berichtet sie etwa, dass sie ,erst viele, viele Jahre später so zufällig erfahren [habe]“ (Vierthaler 2013a, 00:04), dass sie eine jüngere Schwester hatte, die kurz nach der Geburt verstorben war. Die Anreicherung der biographischen Narration durch aus der Akte gewonnene Daten führt mitunter zu einer detaillierteren Informiertheit und äußert sich an ver- 
In das

stbdt. Jugendamt

$\underline{\text { h } 1 \text { er }}$

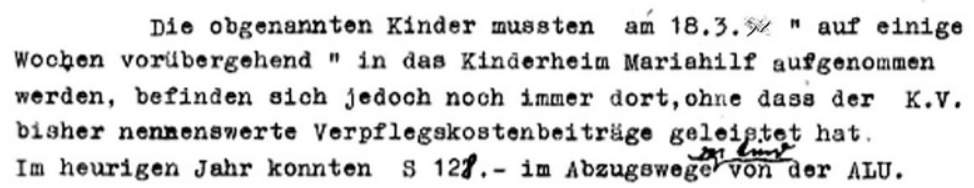

Abb. 2 Ausschnitt aus Jutta Vierthalers Fürsorgeakte (Quelle archiviert am Institut für Erziehungswissenschaft der Universität Innsbruck): Die Akte wird zur Informantin und liefert neue Deutungsangebote

schiedenen Stellen der autobiographischen Erzählung. So etwa in der Schilderung der Heimeinweisung:

[U]nd genau am 18.3. [frühe 1950er Jahre] hat man uns dann ins Kinderheim M. gebracht und unsere Eltern ins Obdachlosenheim. In diesem Kinderheim M. hätten wir eigentlich vorübergehend bleiben sollen und waren dann bis zum August [dreieinhalb Jahre später] tatsächlich dort. [...] Ich sehe auch noch diesen großen Schlafsaal vor mir. [...] Und da hat man uns dann in Gitterbetten gesteckt und meine Mutter hat mir „Die zehn kleinen Negerlein“ vorgelesen. Sie hat mir das alles dann später bestätigt, weil ich habe mir gedacht, ich bilde mir das ein, [aber] es war tatsächlich so. (Vierthaler 2013a, 00:07ff.; siehe hierzu Abb. 2)

Aus dieser Sequenz zur Aufnahme in das Kinderheim wird mehreres deutlich: Jutta Vierthaler weiß nach der Lektüre ihrer Akte nicht nur das genaue Datum der Heimeinweisung, es ist ihr auch wichtig, es im Interview zu nennen. In der zitierten Aussage gibt sie eine weitere Stütze der Erinnerung an: Sie erkundigte sich bei ihrer Mutter, ob ihre Erinnerung zutrifft. Vierthaler spricht in der Passage auch ihre eigene Unsicherheit bezüglich dieser frühen biographischen Erinnerungen an - sie habe gedacht, sie bilde sich das ein -, die sie erfolgreich durch das Nachfragen überwinden konnte. Die Strategie des Rekurses auf schriftlich festgehaltene Daten aus der Akte sowie der Heranziehung einer Gewährsperson, um eigene Erinnerungen $\mathrm{zu}$ validieren und gegenüber der Interviewerin zu plausibilisieren, findet sich an mehreren Stellen im Interview. Das große Bemühen um Genauigkeit und Richtigkeit ihrer Angaben ist vermutlich auch vor dem Hintergrund $\mathrm{zu}$ verstehen, dass die Interviewpartnerin immer wieder erlebte, dass ihre Glaubwürdigkeit in Frage gestellt oder ihr Übertreibung unterstellt wurde. So endet das insgesamt über zweistündige Interview mit der Beschreibung der Reaktionen einiger Leute in ihrem Umfeld:

Du hast nur mehr gehört: [...] ,Mei schau, die wollen alle nur ein Geld [...] und so schlimm kann das ja gar nicht gewesen sein." Und wenn ich dann versucht habe so ansatzweise denen beizubringen, dass es so schlimm war, dann hat einem das niemand geglaubt. (Vierthaler 2013b, 01:56) 


\subsection{Zum Nutzen der Akte als Erinnerungsmobilisator und -mediator}

Wie andere ZeitzeugInnen auch, berichtet Jutta Vierthaler im Interview davon, dass ihr Leben durch eine lange Phase des Schweigens bzw. des Nicht-Erinnerns gekennzeichnet war. Sie selbst deutet dies - wie eingangs bereits ausgeführt - als „Verdrängen“ oder „Davonlaufen“ vor den belastenden Ereignissen der Kindheit: „,ich war immer sehr beschäftigt“ (Vierthaler 2013b, 1:35), erzählt sie. Erst als nach der Matura der Tochter und einer durchgestandenen schweren Erkrankung ihres Partners scheinbar Ruhe in ihr Leben einkehrte, erlebte Jutta Vierthaler eine ,depressive Phase“, wie sie diese selbst nennt, und nahm auf Anraten einer Freundin Kontakt zu einer Therapeutin auf. Im Zuge dieser Therapie wurde ein intensiver Prozess der Erinnerung und Auseinandersetzung mit der eigenen Kindheit in Gang gesetzt, der zum Zeitpunkt des Interviews noch andauerte und, wie die Zeitzeugin vermerkte, durch die Lektüre der Akte noch intensiviert wurde. Die Zeitzeugin war erstaunt darüber, dass sie sich an Ereignisse und Erlebnisse wieder erinnern konnte, die thematisch über die Inhalte der Akte hinausgingen und auch nicht im Zuge ihrer bisherigen Auseinandersetzung mit ihrer Kindheit im Rahmen einer Psychotherapie zu Tage gekommen waren.

An einer Stelle im Interview wird ein solcher Prozess des Neu-Erinnerns besonders deutlich. Jutta Vierthaler erzählt, dass die Kinder im Heim häufig schlechte und nicht ausreichend sättigende Mahlzeiten erhielten, während die Erzieherinnen einen Teil des mit Lebensmittelmarken erworbenen Essens für sich behielten. Sie beginnt sich an eine Episode zu erinnern: Am Nachmittag mussten sich die Kinder eine Weile selbst beschäftigen, damit die Erzieherinnen eine gemeinsame Kaffeepause machen konnten: , ,[...] und ich kann mich erinnern, den Kuchen hast du gerochen bis hinein [in den Aufenthaltsraum]“ (00:43). Einmal sei sie, obwohl das nicht erlaubt war, hinausgerannt, weil sie wissen wollte, was da so gut riecht:

[...] und dann sehe ich den Kuchen da so auf dem Tablett liegen. Ich werde nicht gewusst haben, was Kuchen ist, ich werde nur gerochen [haben], dass das gut schmeckt, nehme ich einmal an, und ich schaue sie so an, die Tante [Erzieherin, Anm.d.V.] und schnuppere so und ich wollte offenbar ein Stück haben (Vierthaler 2013, 00:43).

Stattdessen habe die Erzieherin Jutta Vierthaler in den Aufenthaltsraum zurückgestoßen und dabei sei das Tablett mit dem Kuchen hinuntergefallen, wofür dem Kind die Schuld gegeben wurde. ,[L]ustig, das kommt auch erst jetzt wieder zurück“, resümiert sie diese Sequenz (Vierthaler 2013, 00:44). Der neu in Gang gesetzte Erinnerungsprozess verläuft - wie es die Zeitzeugin im eingangs zitierten Interviewausschnitt auch explizit beschrieben hat - u. a. entlang aufsteigender Bilder. Sie beschreibt, wie sie den Kuchen sieht und führt weiter aus, er habe „so eine goldene Farbe für mich gehabt, das dürfte ein Gugelhupf gewesen sein“, die mit weiteren sinnlichen Wahrnehmungen, hier vor allem Gerüchen nach Kuchen und Kaffee verknüpft sind. Sichtbar wird in dieser Sequenz im Vergleich zu anderen Schilderungen das noch Tentative, nicht vollständig Festgelegte des neu Erinnerten. Das markieren etwa Wendungen wie ,ich nehme an“ oder „offenbar“, aber auch der Wechsel der 


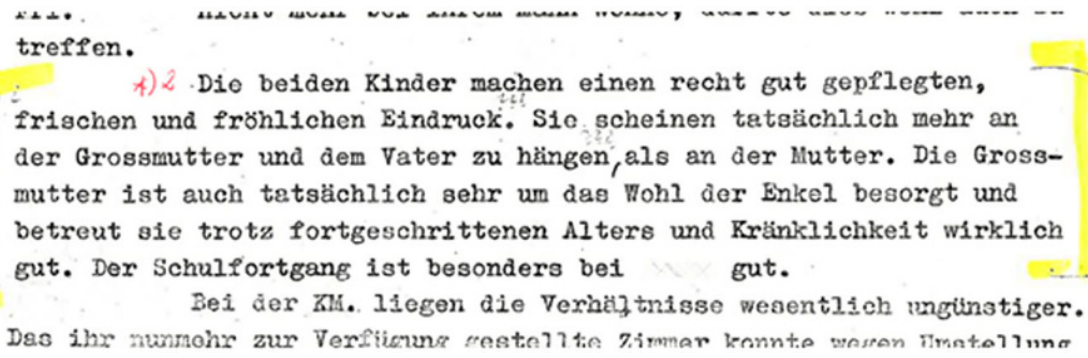

Abb. 3 Ausschnitt aus der Fürsorgeakte Jutta Vierthalers samt nummeriertem Anmerkungssternchen und Hervorhebung durch einen Leuchtstift (Quelle archiviert am Institut für Erziehungswissenschaft der Universität Innsbruck): Die Zeitzeugin tritt mit der Akte in Interaktion

Erzählform in Präsens - ,ich schaue sie so an, die Tante, und schnuppere so“ - und in Futur II - ,ich werde nicht gewusst haben, was Kuchen ist““.

\subsection{Die Akte als Gegenüber: Ein Verhältnis zu den Verhältnissen gewinnen}

Jutta Vierthaler entnahm der Akte nicht nur Informationen und integrierte die durch die Akte ausgelösten Erinnerungen in ihre Lebensgeschichte. Sie trat in eine Auseinandersetzung mit dem Dokument. Den Deutungen der Aktenstückverfasser setzte sie ihre eigenen Deutungen entgegen. Der Akte selbst - die sie vollständig in Kopie besitzt - fügte sie ihre eigenen Anmerkungen hinzu, die Leerstellen benennen oder zum Ausdruck bringen, dass es den JugendamtsmitarbeiterInnen beispielsweise nicht gelang, die Gewalt, der die Kinder im Heim und in der Familie ausgesetzt waren, wahrzunehmen. So ist in einem Aktenvermerk des Jugendamtes zu lesen: „Die beiden Kinder machen einen recht gepflegten, frischen und fröhlichen Eindruck. Sie scheinen tatsächlich mehr an der Grossmutter und dem Vater zu hängen, als an der Mutter" (Akte Vierthaler; vgl. Abb. 3 und 4). Diese Schilderung kontrastiert Frau Vierthaler mit ihrem Kommentar, den sie auf der Rückseite des Blattes notierte:

Da „Besuche“ vom Jugendamt schriftlich angekündigt wurden, wurden mein Bruder und ich entsprechend darauf,,vorbereitet" (Sonntagskleidung, frisch gewaschene Haare, einträchtiges Spielen ... [...]). Außerdem wurde uns permanent damit gedroht, dass wir wieder zurück ins Heim müssten, wenn wir nicht brav sind bzw. der Fürsorgerin etwas anderes, als diese hören sollte, erzählen würden. (Akte Vierthaler)

Im Interview charakterisiert die Zeitzeugin Vater wie Großmutter als autoritär und gewalttätig und beschreibt die schwierige Position, die sie und ihr Bruder in der Familie mit der Stiefmutter und den Halbgeschwistern einnahmen - „fröhlich“ seien sie ihrer Erinnerung nach nicht gewesen. Die Beschreibung des Jugendamtes entspricht demnach nicht der eigenen erinnerten Erfahrung, ähnlich wie es Vierthaler auch bezüglich der eingangs erwähnten Fotografie beschreibt. Ihre Anmerkung zur Aktennotiz kann als Versuch verstanden werden, zu klären, wie diese Fehleinschätzung durch die JugendamtsmitarbeiterInnen, die Kinder seien bei ihrem Vater gut aufgehoben, zustande kam. Gleichzeitig rekonstruiert Jutta Vierthaler damit das als 
Abb. 4 Jutta Vierthalers Kommentar zu der markierten Aktenstelle auf der Rückseite (Quelle archiviert am Institut für Erziehungswissenschaft der Universität Innsbruck): Eine neue Textur entsteht

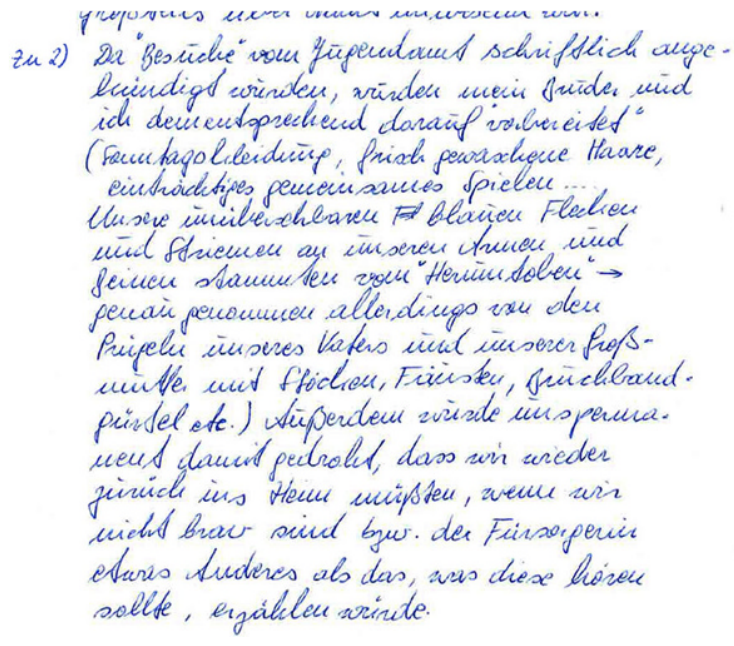

planvoll eingeschätzte Handeln der beteiligten Personen in Bezug auf die jugendamtliche Kontrolle und möglicherweise gerade auch in Hinblick auf die aktenförmige Verwaltung. Denn folgt man der Annahme, dass, was nicht in den Akten ist, auch nicht in der Welt sei, bedeutet dies im Umkehrschluss, dass bloße Einschätzungen durch das schriftliche Festhalten in einer Akte den Status von Tatsachen in der Welt erlangen können - und zwar oft langfristiger als Erinnerungen.

Die Akte erfüllt für Frau Vierthaler die Rolle eines Gegenübers in der Befassung mit der eigenen Vergangenheit. Vor allem auch deshalb, da sämtliche Personen ihrer näheren Familie diesbezüglich nicht zur Verfügung stehen bzw. standen. In der Interaktion mit der Akte konnte die Zeitzeugin einige offene Fragen für sich klären. So erfuhr sie etwa, dass ihr Vater regelmäßig die Zahlung der Unterhaltsbeiträge verabsäumte bis es schließlich zu einer Lohnpfändung kam. Entgegen der im Kinderheim vorgenommenen und für sie wenig plausiblen Unterscheidung in „brave“ und „schlimme“ Kinder als Erklärung dafür, dass manche Kinder ein deutlich höheres Ausmaß an Gewalt erfuhren als andere, erscheint es ihr vor dem Hintergrund der neuen Information wahrscheinlicher, dass dies mit den fehlenden Zahlungen in Zusammenhang stand. Ihrer Erinnerung nach kann sie jedenfalls keine wesentlichen Unterschiede zwischen den sogenannten „Braven“ und „Schlimmen“ ausmachen. Die ,braven“ Kinder, vermutet sie, hatten wohl Eltern, die sich mehr kümmerten und die Unterhaltsbeiträge regelmäßig entrichteten. Unabhängig davon, ob die Interpretation zutrifft oder nicht, gewinnt Frau Vierthaler darüber dass sie sich zu der Akte ins Verhältnis setzt, einen erweiterten Deutungsspielraum, was die Ereignisse ihrer (Heim)Kindheit betrifft. 


\section{Fazit: Akten im Erinnerungs- und Verarbeitungsprozess der Heimgeschichte}

Jutta Vierthalers Lektüre ihrer Fürsorgeakte ist im Kontext ihrer Bemühungen zu verstehen, sich ihre eigene Lebensgeschichte anzueignen. Da ihr viele der üblicherweise vorhandenen Möglichkeiten der Erinnerung an die eigene Kindheit verwehrt blieben, griff sie auf andere zurück: auf Psychotherapie, die Teilnahme an Forschungsprojekten, den Besuch einer Selbsthilfegruppe und auch auf die Lektüre ihrer Akte. Jutta Vierthaler nützt die Akte dabei erstens als Informationsquelle, um ihre Vergangenheit zu rekonstruieren, aber auch um ihr Struktur zu geben und sie mit Sinn zu versehen. Sie verwendet die Akte zweitens als Mobilisierungs- und Einordnungshilfe von Erinnerungen und sie setzt sich schließlich drittens aktiv zur ihr ins Verhältnis: Sie widerspricht, korrigiert, kommentiert. Sie tut dies auch schreibend, sodass aus der ursprünglichen Akte, wie die Abbildungen zeigen, eine neue Textur entsteht: ein neuer, ein weniger fremd verfügter Text erscheint auf der Rückseite der einzelnen Aktenblätter.

Insgesamt zeigt sich am Beispiel Jutta Vierthalers, dass ZeitzeugInnen der Heimgeschichte ihre Akten, die von Jugendämtern oder Erziehungsheimen über sie angefertigt wurden, lesen, interpretieren und als Ressource ihres Erinnerungsprozesses nutzen. Die autobiographischen Erzählungen werden, so zeigt es das Fallbeispiel Jutta Vierthalers deutlich, durch die Kenntnis der eigenen Akte beeinflusst. Dabei legt die Zeitzeugin eine hohe Skepsis gegenüber der Akte als obrigkeitliche Quelle an den Tag: Sie kommentiert einzelne Passagen und versucht damit, die Darstellung der Akte zu korrigieren und sich das Zustandekommen bestimmter Deutungen und damit die getroffenen Entscheidungen sowie die getätigten (oder unterlassenen) Interventionen zu erklären. Durch diese Bearbeitung der eigenen Akte ist es der Zeitzeugin möglich, dem obrigkeitlichen Blick auf ihre Situation als Kind und Jugendliche zu widersprechen - eine Möglichkeit die ihr damals verwehrt blieb. Mit der Bearbeitung der Akte durch die Zeitzeugin ändert sich dies: Sie erhebt Anspruch auf eine Position als legitime Sprecherin. Hatten die Aufzeichnungen durch die MitarbeiterInnen der Jugendämter den Effekt, dass unter Rückgriff auf das in den Akten enthaltene Wissen jugendfürsorgerische Maßnahmen legitimiert werden konnten, so nutzt nun die Zeitzeugin die Möglichkeit, über die Auseinandersetzung mit der Akte und ihre Bearbeitung Ordnung in ihre Vergangenheit zu bringen und das ihr Widerfahrene aufklärbar und plausibel zu machen. Indem Vierthaler infolge der intensiven Auseinandersetzung mit der Akte Inhalte und Deutungen in ihre Lebensgeschichte aufnimmt, andere aber auch zurückweist, gelingt ihr eine Distanznahme sowohl zu den Schilderungen in der Akte als auch zu manchen Erinnerungen. Insgesamt ermöglicht ihr diese durchaus herausfordernde Art der Auseinandersetzung mit der eigenen Akte eine Deutung ihrer Erfahrungen als widerfahrenes Unrecht und als nicht legitime Gewalt.

Anhand des hier vorgestellten Fallbeispiels lassen sich auch Erkenntnisse über Erinnerungs- und Verarbeitungsprozesse der Heimgeschichte im Allgemeinen gewinnen. Für viele ehemalige Heimkinder, die heute als ZeitzeugInnen von ihren Erfahrungen in Erziehungsinstitutionen berichten, ist die Herstellung der eigenen Biographie nur unter erschwerten Bedingungen und mit einer oftmals geringen Aus- 
stattung an Ressourcen möglich. Medien der Erinnerung - wie es auch Akten sein können - sind nicht nur Vermittlungs-, sondern auch Transformationssysteme des Vergangenen. Wie sehr Erinnerungsprozesse von Abwägung, Abgrenzung und Übernahme von Deutungen gekennzeichnet sind bzw. welche Mühen immer wieder auf sich genommen werden müssen, um zu einer Lebensgeschichte, einer Biographie zu gelangen, wird am Einsatz der Akte als „Stütze der Erinnerung“ sichtbar. Für ehemals in Kinder- oder Erziehungsheimen untergebrachte Personen handelt es sich bei den Akten um eine herausfordernde Quelle zur Auseinandersetzung mit der eigenen Lebensgeschichte. Für die Forschung wiederum wirft die durch die Akten ,unterstütze Erinnerung“ der ehemaligen Heimkinder Fragen auf, die mit Erinnern und Vergessen, mit Erinnerungskulturen, Gedächtnispolitiken und ZeitzeugInnenschaften zu tun haben und die in den biografisch-narrativen Interviews mit ehemaligen Heimkindern auf besondere Weise zu Tage treten. Der von uns vorgeschlagene Begriff der ,unterstützten Erinnerung“ spricht demnach ein heuristisches Konzept an, das eine allgemeine Kennzeichnung der Erinnerungsfähigkeit von Individuen ebenso aufgreift, wie es die spezifische Erinnerungskonstellation in ZeitzeugInneninterviews mit Akteneinsatz zu beschreiben im Stande ist. Diese komplexe Gemengelage aus schriftlichem Quellenmaterial und ZeitzeugInnenaussage stellt der Forschung ein Erkenntnismittel bereit, das es erlaubt, über die Funktion von historischem Gedächtnis, persönlicher Erinnerung, Imagination und Repräsentation nachzudenken. Sie reduziert nicht etwa den Wert der mündlichen Quelle, sie vergrößert ihn.

Funding Open access funding provided by University of Innsbruck and Medical University of Innsbruck.

Open Access Dieser Artikel wird unter der Creative Commons Namensnennung 4.0 International Lizenz veröffentlicht, welche die Nutzung, Vervielfältigung, Bearbeitung, Verbreitung und Wiedergabe in jeglichem Medium und Format erlaubt, sofern Sie den/die ursprünglichen Autor(en) und die Quelle ordnungsgemäß nennen, einen Link zur Creative Commons Lizenz beifügen und angeben, ob Änderungen vorgenommen wurden.

Die in diesem Artikel enthaltenen Bilder und sonstiges Drittmaterial unterliegen ebenfalls der genannten Creative Commons Lizenz, sofern sich aus der Abbildungslegende nichts anderes ergibt. Sofern das betreffende Material nicht unter der genannten Creative Commons Lizenz steht und die betreffende Handlung nicht nach gesetzlichen Vorschriften erlaubt ist, ist für die oben aufgeführten Weiterverwendungen des Materials die Einwilligung des jeweiligen Rechteinhabers einzuholen.

Weitere Details zur Lizenz entnehmen Sie bitte der Lizenzinformation auf http://creativecommons.org/ licenses/by/4.0/deed.de.

\section{Literatur}

\section{Verwendete Literatur}

Assmann, Aleida. 1996. Die langen Schatten der Vergangenheit, Erinnerungskultur und Gedächtnispolitik. München: C. H. Beck.

Assmann, Aleida. 2007. Geschichte im Gedächtnis. Von der individuellen Erfahrung zur öffentlichen Inszenierung. München: C. H. Beck.

Assmann, Jan. 2007. Das kulturelle Gedächtnis. Schrift, Erinnerung und politische Identität in frühen Hochkulturen. München: C. H. Beck.

Bereswill, Mechthild, und Peter Rieker. 2017. Call zur Tagung „Multiperspektivische Forschung zur Sozialisation in Institutionen "

Brunner, José. 2012. Medikalisierte Zeugenschaft. In Die Geburt des Zeitzeugen nach 1945, Hrsg. Martin Sabrow, Norbert Freie, 93-110. Göttingen: Wallstein. 
Fivush, Robyn, und Florian Hessel. 2010. Die Entwicklung des autobiographischen Gedächtnisses. In Gedächtnis und Erinnerung: Ein interdisziplinäres Handbuch, Hrsg. Christian Gudehus, Ariane Eichenberg, und Harald Welzer, 45-53. Stuttgart: Metzler.

Flick, Uwe. 2009. Qualitative Sozialforschung. Eine Einführung, 2. Aufl., Reinbeck: Rowohlt.

Foucault, Michel. 1976. Überwachen und Strafen. Die Geburt des Gefängnisses. Frankfurt a.M.: Suhrkamp.

Guerrini, Flavia. 2018. Öffentliche Fürsorge und ihre Effekte. Zur Subjektbildung in der historischen Jugendfürsorge und Heimerziehung

Halbwachs, Maurice. 1985. Das Gedächtnis und seine sozialen Bedingungen. Frankfurt a.M.: Suhrkamp. 1925.

Heimkinder reden. 2015. Jetzt reden wir! Ehemalige Heimkinder erzählen. https://www.heimkinder-reden. at/. Zugegriffen: 11. Juni 2019.

Hönigsberger, Georg, und Irmtraut Karlsson. 2013. Verwaltete Kindheit. Der österreichische Heimskandal. Berndorf: Kral.

Kuhlmann, Carola. 2008. So erzieht man keinen Menschen! Lebens- und Berufserinnerungen aus der Heimerziehung der 50er und 60er Jahre. Wiesbaden: VS

Lucius-Hoene, Gabriele, und Arnulf Deppermann. 2004. Rekonstruktion narrativer Identität. Ein Arbeitsbuch zur Analyse narrativer Interviews. Wiesbaden: VS.

Müller, Siegfried, und Hartmut Müller. 1984. Akten/Aktenanalysen. In Handbuch zur Sozialarbeit, Sozialpädagogik, Hrsg. Eyferth Hanns, 23-42. Neuwied: Luchterhand.

Niethammer, Lutz. 1980. Lebenserfahrung und kollektives Gedächtnis. Die Praxis des „Oral History“. Frankfurt a.M.: Suhrkamp.

Ralser, Michaela. 2010. Das Subjekt der Normalität. Das Wissensarchiv der Psychiatrie: Kulturen der Krankheit um 1900. München: Wilhelm Fink.

Ralser, Michaela, Anneliese Bechter, und Flavia Guerrini. 2014. Regime der Fürsorge. Eine Vorstudie zur Geschichte der Tiroler und Vorarlberger Erziehungsheime und Fürsorgeerziehungssysteme der Zweiten Republik. Innsbruck: iup.

Ralser, Michaela, Nora Bischoff, Flavia Guerrini, Christine Jost, Ulrich Leitner, und Martina Reiterer. 2017. Heimkindheiten. Geschichte der Jugendfürsorge und Heimerziehung in Tirol und Vorarlberg. Innsbruck, Wien, Bozen: StudienVerlag.

Ramsauer, Nadja. 2000. „Verwahrlost“. Kindeswegnahmen und die Entstehung der Jugendfürsorge im schweizerischen Sozialstaat 1900-1945. Zürich: Chronos.

Rosenthal, Gabriele. 2008. Interpretative Sozialforschung. Eine Einführung. Weinheim: Juventa.

Sabrow, Martin. 2012. Der Zeitzeuge als Wanderer zwischen den Zeiten. In Die Geburt des Zeitzeugen nach 1945, Hrsg. Martin Sabrow, Norbert Freie, 13-33. Göttingen: Wallstein.

Sabrow, Martin, et al. 2007. Wohin treibt die DDR-Erinnerung? Dokumentation einer Debatte. Göttingen: Vandenhoeck \& Ruprecht.

Schreiber, Horst. 2010. Im Namen der Ordnung. Heimerziehung in Tirol. Innsbruck, Wien, Bozen: StudienVerlag.

Schütze, Fritz. 1983. Biographieforschung und narratives Interview. Neue Praxis 13(3):283-293.

Sieder, Reinhard, und Andrea Smioski. 2012. Der Kindheit beraubt. Gewalt in den Erziehungsheimen der Stadt Wien. Innsbruck, Wien, Bozen: StudienVerlag.

Universität Innsbruck. 2019. Heimgeschichte. https://www.uibk.ac.at/iezw/heimgeschichteforschung. Zugegriffen: 11. Juni 2019.

Wierling, Dorothee. 2003. Oral History. In Aufriss der Historischen Wissenschaften, Bd. 7, Hrsg. Michael Maurer, 81-151. Stuttgart: Reclam.

Zaft, Matthias. 2011. Der erzählte Zögling. Narrative in den Akten der deutschen Fürsorgeerziehung. Bielefeld: transcript.

\section{Quellen}

Vierthaler, Jutta (Pseudonym). 2013. Interview geführt am 25.09.2013, zwei Audiodateien (a u. b), archiviert am Institut für Erziehungswissenschaft der Universität Innsbruck.

Vierthaler, Jutta. Fürsorgeakte, Stadtarchiv Innsbruck (StAI), Bestand Sozialakten - Jugendwohlfahrt, Jugendfürsorgeakte 69-BS, aa3/1951, in Kopie überlassen von der Protagonistin und archiviert am Institut für Erziehungswissenschaft der Universität Innsbruck.

Vierthaler, Jutta. Datensammlung: Daten/Zusammenfassung zu den diversen Schriftstücken, überlassen und archiviert am Institut für Erziehungswissenschaft der Universität Innsbruck. 